\title{
Homomorphisms between Fuzzy Approximation Spaces Based on Residuated Lattice
}

\author{
Yuan Zhao \\ College of Mathematics and Statistics, Zhengzhou Normal University, Zhengzhou 450052, China \\ Correspondence should be addressed to Yuan Zhao; zhld.ls@gmail.com
}

Received 13 September 2013; Accepted 16 January 2014; Published 3 March 2014

Academic Editor: Kazutake Komori

Copyright (C) 2014 Yuan Zhao. This is an open access article distributed under the Creative Commons Attribution License, which permits unrestricted use, distribution, and reproduction in any medium, provided the original work is properly cited.

Two kinds of homomorphisms of fuzzy approximation spaces based on complete residuated lattice are proposed. The homomorphisms are structure-preserving maps in some sense. We also introduce the fuzzy approximation subspaces and investigate their correspondence with the homomorphisms. Given a fuzzy equivalence relation, the factor set of the fuzzy approximation space is discussed.

\section{Introduction}

The rough set theory proposed by Pawlak $[1,2]$ is a mathematical tool to deal with intelligent systems characterized by insufficient and incomplete information. Its key concept is a pair of lower and upper approximation operators based on an equivalence relation. Through the pair of operators, the hidden information may be unraveled. Rough set theory has received much attention in the past decades. The successful applications of rough set theory in a variety of problems have fully demonstrated the usefulness of rough set theory.

In Pawlak's rough set theory, equivalence relation is an important concept. The lower and upper approximation operators are built from the equivalence classes. By replacing equivalence relations with arbitrary binary relations, generalizations of Pawlak's rough set have been obtained. Fuzzy sets provide a convenient tool for representing vague concepts by allowing partial memberships. So by replacing the binary relations with fuzzy ones, various kinds of fuzzy rough sets could be obtained [3-10]. As in classical rough set theory, there are two approaches [11] to deal with the fuzzy case, named the constructive and axiomatic ones. Fuzzy rough sets based on residuated lattice were proposed by Radzikowska and Kerre in [12]. She and Wang discussed the axiomatic approach of the fuzzy rough sets based on residuated lattice in [13].

Sometimes it is necessary to construct the connection of two approximation spaces, in order to transmit information hidden in them. And also we want to deal with a small database rather than a large one, so building the communication between them is very important. The homomorphisms between rough sets are proposed by Graymala-Busse in [14], in order to deal with the correspondence between two information systems. It is very useful in dealing with data compression in information systems and approximation spaces. In $[15,16]$, Wang et al. proposed the type 1 and type 2 consistent functions, then introduced the homomorphisms in terms of the consistent function of information systems and the fuzzy information systems, respectively. Then Zhu and Wen revisited the homomorphisms between information systems (classical and fuzzy case, resp.) in $[17,18]$. And the predecessorconsistent function and successor-consistent function have been introduced, they are just the type 1 and type 2 consistent functions in $[17,18]$. The researchers explored more about invariant properties under the homomorphisms in the literature.

In this paper, we discuss the homomorphisms between fuzzy rough approximation spaces based on complete residuated lattice. We propose the homomorphisms between them as structure preserving maps and discuss their properties. The fuzzy approximation subspaces and the factor sets of fuzzy approximation spaces are introduced. The relationship between the factor sets and the homomorphisms is also investigated.

We focus on the homomorphism between fuzzy approximation spaces in this paper. It is organized as follows. In 
Section 2, we recall some fundamental properties of residuated lattice, $L$-fuzzy sets and $L$-fuzzy relations. Some basic properties of $L$-fuzzy rough sets are also given in Section 2. In Section 3, we introduce the Z-homomorphisms induced by Zadeh's forward operators and discuss their properties. The fuzzy approximation subspaces are proposed in Section 4 and the correspondence with the homomorphisms is also discussed. In Section 5, we propose another kind of homomorphisms that relies more heavily on the relations of the two fuzzy approximation spaces. The factor set of fuzzy approximation spaces is also introduced in this section. Section 6 carries the conclusions.

\section{Preliminaries}

A residuated lattice $[19,20](L, *, \rightarrow, \vee, \wedge, 0,1)$ is an algebra with two binary operations $*, \rightarrow$ on $L$ such that

(1) $(L, \vee, \wedge, 0,1)$ is a bounded lattice with the greatest element 1 and the least element 0 ;

(2) $(L, *, 1)$ is a commutative monoid and $*$ is isotonic at both arguments;

(3) $(*, \rightarrow)$ are adjoint pairs; that is, $x * y \leq z$ if and only if $x \leq y \rightarrow z$ for all $x, y, z \in L$.

A residuated lattice is said to be complete if the underlying lattice is complete.

In what follows, $*$ is sometimes called a generalized triangular norm and the implicator $\rightarrow$ is called the residuum of *. An implicator $I$ is called left monotonic (resp., right monotonic) if $I(\cdot, a)$ is decreasing for every $a \in L$ (resp., $I(a, \cdot)$ is increasing). If $I$ is both left and right monotonic, it is called hybrid monotonic.

In the paper, $L$ always denotes a complete residuated lattice if not otherwise specified. The precomplement on $L$ is the map $\neg: L \rightarrow L$ defined by $\neg a=a \rightarrow 0$ for every $a \in L$. Some basic properties of complete residuated lattices are as follows (more properties about complete residuated lattices can be referred to in $[19,20])$ :

(1) $1 \rightarrow a=a, a \leq b \Leftrightarrow a \rightarrow b=1$;

(2) $(a \rightarrow b) *(b \rightarrow c) \leq a \rightarrow c,(a \rightarrow b) *(c \rightarrow d) \leq$ $(a * c) \rightarrow(b * d)$

(3) $a \rightarrow(b \rightarrow c)=b \rightarrow(a \rightarrow c)=(a * b) \rightarrow c$;

(4) $a \leq(b \rightarrow a * b), a *(a \rightarrow b) \leq b$;

(5) $a *\left(\bigvee_{i \in I} b_{i}\right)=\bigvee_{i \in I}\left(a * b_{i}\right)$;

$(6) a \rightarrow\left(\bigwedge_{i \in I} b_{i}\right)=\bigwedge_{i \in I}\left(a \rightarrow b_{i}\right),\left(\bigvee_{i \in I} a_{i}\right) \rightarrow b=$ $\bigwedge_{i \in I}(a \rightarrow b)$

(7) $a \rightarrow(b \rightarrow a)=1, a \rightarrow(b \rightarrow a * b)=1$;

(8) $b \rightarrow c \leq(a \rightarrow b) \rightarrow(a \rightarrow c), c \rightarrow b \leq(b \rightarrow$ a) $\rightarrow(c \rightarrow a)$

(9) $a \leq \neg \neg a$;

(10) $a \rightarrow \neg b=\neg(a * b) ; \neg\left(\bigvee_{i \in I} a_{i}\right)=\bigwedge_{i \in I}\left(\neg a_{i}\right)$
If $\neg \neg a=a$ holds for every $a \in L$, then $L$ is called involutive. In this case, the following conditions hold:

(11) $a \rightarrow b=(\neg b) \rightarrow(\neg a)=\neg(a *(\neg b)) ; a * b=\neg(a \rightarrow$ $(\neg b))$;

(12) $\neg\left(\bigwedge_{i \in I} a_{i}\right)=\bigvee_{i \in I}\left(\neg a_{i}\right)$.

When the $*$ operator is exactly the operator $\wedge$ of the residuated lattice, it is called Heyting algebra. A complete Heyting algebra is a special case of complete residuated lattice, it is also called frame.

In [21], Goguen first introduced $L$-fuzzy set as a generalization of Zadeh's-fuzzy set, where $L$ is a complete residuated lattice. An $L$-fuzzy set $A$ on $X$ is a map $A: X \rightarrow L$ and all the $L$-fuzzy sets on $U$ are denoted by $L^{X}$. For every $a \in L$, we use $\bar{a}$ to denote the constant $L$-fuzzy set on $X$. For $A, B \in L^{X}$, we denote $A \subseteq B$ if $A(x) \leq B(x)$ for every $x \in X$.

Given two $L$-fuzzy sets $A$ and $B$, new $L$-fuzzy sets can be induced as follows:

$$
\begin{aligned}
& (A * B)(x)=A(x) * B(x) ; \\
& (A \wedge B)(x)=A(x) \wedge B(x) ; \\
& (A \vee B)(x)=A(x) \vee B(x) ; \\
& (A \rightarrow B)(x)=A(x) \rightarrow B(x) ; \\
& (\neg A)(x)=A(x) \rightarrow 0 .
\end{aligned}
$$

In [22-27], various types of reflexivities, antisymmetries, and transitivities are investigated. According to these notions, proximity and similarity fuzzy relations, fuzzy partitions, and fuzzy orderings, fuzzy equivalences are introduced. Properties of these fuzzy binary relations and operations on them are also studied.

An $L$-fuzzy relation [21] $R$ on $X$ is a map $R: X \times X \rightarrow L$, where $R(x, y)$ denotes the degree between $x$ and $y,(x, y) \in$ $U \times U$.

$R$ is serial if for every $x \in X, \bigvee_{y \in X} R(x, y)=1 ; R$ is reflexive if $1 \leq R(x, x)$ for all $x \in X ; R$ is transitive if $\bigvee_{y \in X} R(x, y) * R(y, z) \leq R(x, z)$ for all $x, z \in X ; R$ is symmetric if $R(x, y)=R(y, x)$ for all $x, y \in X ; R$ is antisymmetric if for all $x, y \in X, R(x, y)=R(y, x)=1$ implies that $x=y$.

A reflexive, symmetric, and transitive $L$-fuzzy relation $R$ on a set $X$ is called a fuzzy equivalence relation [28]. The antisymmetric fuzzy equivalence relation is called a fuzzy equality. The set of fuzzy equivalence relations on $X$ forms a complete lattice. The meet coincides with the intersection of the $L$-fuzzy relations, but the join does not coincide with the ordinary union.

Let $R$ be a fuzzy equivalence relation on a set $X$. For every $a \in X, R_{a}$ is called a fuzzy equivalence class determined by $R$, where $R_{a}(y)=R(a, y)$ for every $y \in X$. The set $X / R=$ $\left\{R_{a}, a \in X\right\}$ is called the factor set of $X$ with respect to $R$.

Lemma 1 (see [28]). Let $R$ be a fuzzy equivalence relation on a set $X$. Then, for every $x, y \in X$, the following is true:

(1) $R(x, y)=\bigvee_{z \in X} R(x, z) * R(y, z)$;

(2) $R(x, y)=1 \Leftrightarrow R_{x}=R_{y}$. 
The notion of fuzzy rough sets based on residuated lattice was proposed by Radzikowska and Kerre in [12]. By taking complete residuated lattices instead of $[0,1]$ as the truth value structures, it differs from the concept of fuzzy rough sets that are investigated in [4-10].

Definition 2 (see $[12,13])$. Let $(L, *, \rightarrow, \vee, \wedge, 0,1)$ be a complete residuated lattice, $U$ a nonempty set, and $R$ an $L$-relation on $U$. $(U, R)$ is called an $L$-fuzzy approximation space. For every $A \in L^{U}$ and every $x \in U, \bar{R}(A)$ and $\underline{R}(A)$ are defined as

$$
\begin{gathered}
\bar{R}(A)(x)=\bigvee_{y \in U}(R(x, y) * A(y)), \\
\underline{R}(A)(x)=\bigwedge_{y \in U}(R(x, y) \longrightarrow A(y)) .
\end{gathered}
$$

$\bar{R}(A)$ and $\underline{R}(A)$ are called an upper and lower $L$ fuzzy rough approximation of $A$, respectively. The pair $(\underline{R}(A), \bar{R}(A))$ is called the fuzzy rough set of $A$; if $\underline{R}(A)=A=$ $\bar{R}(A)$, then $A$ is called $R$-definable fuzzy set.

It is easy to check that fuzzy rough set defined above is a wide generalization of $(I, T)$-fuzzy rough sets [10], where $T$ is a $t$-norm and $I$ is the residual implicator based on $T$. In the case when $L=\{0,1\}, A$ and $R$ can be reduced to crisp subsets of $U$ and $U \times U$, respectively, and $\bar{R}(A)$ and $\underline{R}(A)$ are precisely the corresponding concepts in classical rough set theory. Nevertheless, $\underline{R}(A) \subseteq \bar{R}(A)$, which is true in the classical case, is not true in the fuzzy setting.

The following proposition provides basic properties of the lower and upper $L$-fuzzy rough approximation operators.

Proposition 3 (see $[12,13])$. Let $(L, *, \rightarrow, \vee, \wedge, 0,1)$ be a complete residuated lattice and $(U, R)$ an $L$-fuzzy approximation space. Then for all $A, B \in L^{U}$ and $A_{i} \in L^{U}$ (for all $i \in I$ ),

(1) $\bar{R}(\emptyset)=\emptyset, \underline{R}(U)=U$;

(2) If $A \subseteq B$, then $\underline{R}(A) \subseteq \underline{R}(B), \bar{R}(A) \subseteq \bar{R}(B)$;

(3) $\underline{R}(A) \subseteq \neg \bar{R}(\neg A), \bar{R}(A) \subseteq \neg \underline{R}(\neg A), \neg \bar{R}(A)=\underline{R}(\neg A)$;

(4) $\bar{R}\left(\bigvee_{i \in I} A_{i}\right)=\bigvee_{i \in I} \bar{R}\left(A_{i}\right), \underline{R}\left(\bigwedge_{i \in I}\left(A_{i}\right)\right)=\bigwedge_{i \in I} \underline{R}\left(A_{i}\right)$;

(5) $\bar{R}(\bar{a} * A)=\bar{a} * \bar{R}(A), \underline{R}(\bar{a} \rightarrow A)=\bar{a} \rightarrow \underline{R}(A)$;

(6) $\bar{R}(\bar{a}) \subseteq \bar{a}, \bar{a} \subseteq \underline{R}(\bar{a})$;

(7) $\bar{R}(\bar{a})=\bar{a} \Leftrightarrow \bar{R}(U)=U, \bar{a}=\underline{R}(\bar{a}) \Rightarrow \underline{R}(\emptyset)=\emptyset$;

(8) $\bar{R}\left(1_{y} * \bar{a}\right)(x)=R(x, y) * \bar{a}, \underline{R}\left(1_{y} \rightarrow \bar{a}\right)=R(x, y) \rightarrow$ $\bar{a}$, where $1_{y} \in L^{U}$, defined by

$$
1_{y}(x)= \begin{cases}1, & \text { if } x=y \\ 0, & \text { otherwise }\end{cases}
$$

It is necessary to mention that when $L$ is not involutive, $\underline{R}(A)$ and $\bar{R}(A)$ are not dual to each other. So many results of $\underline{R}(A)$ based on complete residuated lattice are not dual to $\bar{R}(A)$ as in many other kinds of fuzzy rough sets. However, the following proposition shows that $\underline{R}(A)$ and $\bar{R}(A)$ are dual to each other when the truth value is an involutive complete residuated lattice.
Proposition 4 (see $[13])$. Let $(L, *, \rightarrow, \vee, \wedge, 0,1)$ be an involutive complete residuated lattice and $(U, R)$ an $L$-fuzzy approximation space. Then for every $A \in L^{U}, \underline{R}(A)=\neg \bar{R}(\neg A)$, $\bar{R}(A)=\neg \underline{R}(\neg A)$.

For more details about fuzzy rough sets based on residuated lattices, please refer to $[12,13]$. In the following, we use fuzzy instead of $L$-fuzzy for the consistency of the context.

\section{Homomorphism Determined by Zadeh's Fuzzy Forward Operators}

In this section, we always assume that $L$ is a complete residuated lattice. Let $f: X \rightarrow Y$ be a map, the Zadeh's fuzzy forward operators $f_{L} \rightarrow L^{X} \rightarrow L^{Y}$ and fuzzy backward operators $f_{L}^{\leftarrow}: L^{Y} \rightarrow L^{X}$ are defined as follows:

$$
\begin{gathered}
f_{L}^{\rightarrow}(A)(a)=\bigvee_{f(x)=a} A(x), \quad \forall A \in L^{X}, \forall a \in Y ; \\
f_{L}^{\leftarrow}(B)(x)=B(f(x)), \quad \forall B \in L^{Y}, \forall x \in X .
\end{gathered}
$$

In this section, we proposed the Z-homomorphisms determined by Zadeh's forward operators between fuzzy approximation spaces based on complete residuated lattice. Some of their properties are discussed.

Definition 5. Let $\left(X, R_{1}\right),\left(Y, R_{2}\right)$ be fuzzy approximation spaces and $f: X \rightarrow Y$ a map. Then $f$ is called an upper Z-homomorphism if it satisfies $f \rightarrow\left(\overline{R_{1}}(A)\right) \subseteq \overline{R_{2}}(f \rightarrow(A))$, for every $A \in L^{X} ; f$ is called a lower Z-homomorphism if it satisfies $f^{\rightarrow}\left(R_{1}(A)\right) \subseteq R_{2}\left(f^{\rightarrow}(A)\right)$, for every $A \in L^{X} ; f$ is called a Z-homomorphism if it is both fuzzy upper and fuzzy lower Z-homomorphism.

We call a map $f:\left(X, R_{1}\right) \rightarrow\left(Y, R_{2}\right)$ relation-preserving if and only if $R_{1}(x, y) \leq R_{2}(f(x), f(y))$; $f$ is called relationembedding if $R_{1}(x, y)=R_{2}(f(x), f(y))$; $f$ is called relationisomorphism if $f$ is bijective and relation-embedding. For every $y \in X, 1_{y}$ denotes the fuzzy subset of $X$, where

$$
1_{y}(x)= \begin{cases}1, & \text { if } y=x \\ 0, & \text { otherwise }\end{cases}
$$

Theorem 6. Let $\left(X, R_{1}\right),\left(Y, R_{2}\right)$ be fuzzy approximation spaces and $f: X \rightarrow Y$ a map. Then $f$ is an upper $Z$ homomorphism if and only if $f$ is relation-preserving.

Proof. For every $x, y \in X$,

$$
\begin{aligned}
f \rightarrow\left(\overline{R_{1}}\left(1_{y}\right)\right)(f(x)) & =\bigvee_{f(z)=f(x)} \overline{R_{1}}\left(1_{y}\right)(z) \\
& =\bigvee_{f(z)=f(x)} \bigvee_{u \in X} R_{1}(z, u) * 1_{y}(u) \\
& =\bigvee_{f(z)=f(x)} R_{1}(z, y) ;
\end{aligned}
$$




$$
\begin{aligned}
\overline{R_{2}}(f \rightarrow)\left(1_{y}\right)(f(x)) & =\bigvee_{a \in Y} R_{2}(f(x), a) *\left(\bigvee_{f(z)=a} 1_{y}(z)\right), \\
& =\bigvee_{a \in Y} \bigvee_{f(z)=a} R_{2}(f(x), a) * 1_{y}(z) \\
& =R_{2}(f(x), f(y)) .
\end{aligned}
$$

Since $f$ is an upper Z-homomorphism, we have $R_{1}(x, y) \leq$ $R_{2}(f(x), f(y))$; that is, $f$ is relation-preserving.

On the other hand, for every $A \in L^{X}, a \in Y$,

$$
\begin{aligned}
& f^{\rightarrow}\left(\overline{R_{1}}(A)\right)(a)=\bigvee_{f(z)=a}\left(\bigvee_{u \in X} R_{1}(z, u) * A(u)\right) \\
& \overline{R_{2}}(f \rightarrow(A))(a)=\bigvee_{b \in Y} R_{2}(a, b) *\left(\bigvee_{f(z)=a} A(z)\right) .
\end{aligned}
$$

Since $f:\left(X, R_{1}\right) \rightarrow\left(Y, R_{2}\right)$ is a relation-preserving map, we have $R_{1}(z, u) \leq R_{2}(f(z), f(u))=R_{2}(a, f(u))$, for $z \in X$ such that $f(z)=a$. So $R_{1}(z, u) * A(u) \leq R_{2}(a, f(u)) *$ $\left(\bigvee_{f(v)=f(u)} A(v)\right)$. Hence,

$$
\begin{aligned}
\bigvee_{u \in X} R_{1}(z, u) * A(u) & \leq \bigvee_{u \in X} R_{2}(a, f(u)) *\left(\bigvee_{f(v)=f(u)} A(v)\right) \\
& \leq \bigvee_{b \in Y} R_{2}(a, b) *\left(\bigvee_{f(v)=b} A(v)\right)
\end{aligned}
$$

Therefore, $f \rightarrow\left(\overline{R_{1}}(A)\right) \subseteq \overline{R_{2}}\left(f^{\rightarrow}\right)(A)$.

Corollary 7. Let $\left(X, R_{1}\right),\left(Y, R_{2}\right)$ be fuzzy approximation spaces and $f: X \rightarrow Y$ a map. Then $f$ is an upper $Z$ homomorphism if and only if $f \rightarrow\left(\overline{R_{1}}\left(1_{x}\right)\right) \subseteq \overline{R_{2}}\left(f^{\rightarrow}\right)\left(1_{x}\right)$ for every $x \in X$.

Proof. The claim is easily derived from Theorem 6.

Corollary 8. Let $\left(X, R_{1}\right),\left(Y, R_{2}\right)$ be fuzzy approximation spaces and $f: X \rightarrow Y$ a surjective upper $Z$-homomorphism. Then the following assertions hold:

(1) If $R_{1}$ is serial, then $R_{2}$ is serial;

(2) If $R_{1}$ is reflexive, then $R_{2}$ is reflexive.

Proof. Since $f$ is surjective, for every $a \in Y$, there exists a $x \in X$, such that $f(x)=a$.

(1) Assume that $R_{1}$ is serial, we have

$$
1=\bigvee_{y \in X} R_{1}(x, y) \leq \bigvee_{y \in X} R_{2}(a, f(y)) \leq \bigvee_{b \in Y} R_{2}(a, b) .
$$

Hence, $R_{2}$ is serial.

(2) $R_{2}(a, a) \geq R_{1}(x, x)=1$, so we have $R_{2}$ that is reflexive.
Proposition 9. Let $\left(X, R_{1}\right),\left(Y, R_{2}\right)$ be fuzzy approximation spaces and $f: X \rightarrow Y$ a lower $Z$-homomorphism. If $R_{1}, R_{2}$ are reflexive and transitive, then $f^{\rightarrow}\left(\underline{R_{1}}\left(R_{1 x}\right)\right)=\underline{R_{2}}\left(f^{\rightarrow}\left(R_{1 x}\right)\right)$.

Proof. For every $x \in X, a \in Y, R_{1}, R_{2}$ is reflexive and transitive as follows:

$$
\begin{aligned}
f^{\rightarrow}\left(\underline{R_{1}}\left(R_{1 x}\right)\right)(a) & =\bigvee_{f(z)=a}\left(\bigwedge_{u \in X} R_{1}(z, u) \longrightarrow R_{1 x}(u)\right) \\
& =\bigvee_{f(z)=a} R_{1}(x, z), \\
\underline{R_{2}}\left(f \rightarrow\left(R_{1 x}\right)\right)(a) & =\bigwedge_{b \in Y} R_{2}(a, b) \longrightarrow\left(\bigvee_{f(z)=b} R_{1}(x, z)\right) .
\end{aligned}
$$

Since $f$ is a lower Z-homomorphism, we have $\bigvee_{f(z)=a} R_{1}(x$, $z) \leq \bigwedge_{b \in Y} R_{2}(a, b) \rightarrow\left(\bigvee_{f(z)=b} R_{1}(x, z)\right) \leq \bigvee_{f(z)=a} R_{1}(x, z)$. Therefore, $f \rightarrow\left(R_{1}\left(R_{1 x}\right)\right)=\underline{R_{2}}\left(f \rightarrow\left(R_{1 x}\right)\right)$.

Theorem 10. Let $L$ be an involutive complete residuated lattice, $\left(X, R_{1}\right),\left(Y, R_{2}\right)$ be fuzzy approximation spaces, and $f: X \rightarrow$ $Y$ be bijective. Then the following assertions hold:

(1) $f$ is an upper Z-homomorphism if and only if $f^{\rightarrow}\left(\underline{R_{1}}(A)\right) \supseteq \underline{R_{2}}\left(f^{\rightarrow}(A)\right)$;

(2) $f$ is a lower Z-homomorphism if and only if $f^{\rightarrow}\left(\overline{R_{1}}(A)\right) \supseteq \overline{R_{2}}(f \rightarrow(A))$;

(3) $f$ is a Z-homomorphism if and only if $f^{\rightarrow}\left(\overline{R_{1}}(A)\right)=$ $\overline{R_{2}}(f \rightarrow(A))$ and $f \rightarrow\left(R_{1}(A)\right)=R_{2}(f \rightarrow(A))$. Moreover, $R_{1}(x, y)=R_{2}(f(\bar{x}), f(y))$.

Proof. (1) Since $f$ is bijective, there exists a unique $x \in X$ such that $f(x)=a$ for every $a \in Y$. So for every $A \in L^{X}$, we have $f \rightarrow(\neg A)=\neg f \rightarrow(A)$. Therefore, $f$ is an upper Zhomomorphism $\Leftrightarrow f^{\rightarrow}\left(\overline{R_{1}}(A)\right) \subseteq \overline{R_{2}}\left(f^{\rightarrow}(A)\right) \Leftrightarrow$

$$
\begin{aligned}
f^{\rightarrow} & \left(\overline{R_{1}}(\neg A)\right) \subseteq \overline{R_{2}}(f \rightarrow(\neg A)) \\
& \Longleftrightarrow f \rightarrow\left(\neg \underline{R_{1}}(A)\right) \subseteq \neg\left(\underline{R_{2}}(f \rightarrow(A))\right) \\
& \Longleftrightarrow \neg\left(f^{\rightarrow}\left(\underline{R_{1}}(A)\right)\right) \subseteq \neg\left(\underline{R_{2}}\left(f^{\rightarrow}(A)\right)\right) \\
& \Longleftrightarrow f \rightarrow\left(\underline{R_{1}}(A)\right) \supseteq \underline{R_{2}}(f \rightarrow(A)) .
\end{aligned}
$$

The duality of $\underline{R}$ and $\bar{R}$ is guaranteed when $L$ is involutive.

(2) It could be proved similarly as (1).

(3) It follows (1), (2), and Definition 5. From Theorem 6, we have $R_{1}(x, y)=R_{2}(f(x), f(y))$.

Proposition 11. If $L$ is not involutive, $f:\left(X, R_{1}\right) \rightarrow\left(Y, R_{2}\right)$ is a bijective Z-homomorphism, we also have $f^{\rightarrow}\left(R_{1}(A)\right)=$ $\underline{R_{2}}(f \rightarrow(A))$. 
Proof. Since $f$ is bijective, for every $a \in Y$, there exists a unique $x \in X$ such that $f(x)=a$. So for every $A \in L^{X}$,

$$
\begin{aligned}
& \left.f^{\rightarrow} \underline{\left(R_{1}\right.}(A)\right)(f(x))=\bigwedge_{y \in X} R_{1}(x, y) \longrightarrow A(y), \\
& \underline{R_{2}}(f \rightarrow(A))(f(x))=\bigwedge_{y \in X} R_{2}(f(x), f(y)) \longrightarrow A(y) .
\end{aligned}
$$

Because $f$ is a homomorphism, we have $f \rightarrow\left(R_{1}(A)\right) \subseteq$ $R_{2}(f \rightarrow(A))$ and $f$ is relation-preserving. Therefore, $R_{1}(x$, $\overline{y)} \rightarrow A(y) \geq R_{2}(f(x), f(y)) \rightarrow A(y)$. So $f \rightarrow\left(R_{1}(A)\right) \supseteq$ $\underline{R_{2}}(f \rightarrow(A))$.

Proposition 12. Let $L$ be an involutive complete residuated lattice, $\left(X, R_{1}\right),\left(Y, R_{2}\right)$ be fuzzy approximation spaces, and $f$ : $X \rightarrow Y$ be a bijective map. Then $f$ is a Z-homomorphism if and only if $f^{-1}$ is a Z-homomorphism.

Proof. Assume that $f$ is a bijective Z-homomorphism, for every $B \in L^{Y}$, we have

$$
\begin{aligned}
\left(f^{-1}\right) \rightarrow\left(\overline{R_{2}}(B)\right)(x) & =\bigvee_{y \in X} R_{2}(f(x), f(y)) * B(f(y)) \\
& =\overline{R_{2}}(f \rightarrow(B \circ f))(f(x)) \\
\overline{R_{1}}\left(\left(f^{-1}\right) \rightarrow(B)\right)(x) & =\bigvee_{y \in X} R_{1}(x, y) *(B \circ f)(y) \\
& =f \rightarrow\left(\overline{R_{1}}(B \circ f)\right)(f(x)) .
\end{aligned}
$$

From Theorem 10, $\left(f^{-1}\right) \rightarrow\left(\overline{R_{2}}(B)\right)=\overline{R_{1}}\left(\left(f^{-1}\right) \rightarrow(B)\right)$. We can also prove that $\left(f^{-1}\right) \rightarrow\left(R_{2}(B)\right)=R_{1}\left(\left(f^{-1}\right) \rightarrow(B)\right)$ via direct computing. Therefore, $\overline{f^{-1}}$ is a Z-homomorphism. The converse could be proved similarly.

Remark 13. Assume that $L$ is not involutive, and $\left(X, R_{1}\right)$, $\left(Y, R_{2}\right)$ are fuzzy approximation spaces.

(1) $f:\left(X, R_{1}\right) \rightarrow\left(Y, R_{2}\right)$ is a bijective Z-homomorphism, we could not deduce that $f^{-1}$ is a bijective $Z$ homomorphism in general.

(2) If $f$ and $f^{-1}$ are both bijective upper Z-homomorphisms, then $R_{1}(x, y)=R_{2}(f(x), f(y))$. Certainly, if $f$ and $f^{-1}$ are both bijective Z-homomorphism, we also have $R_{1}(x, y)=R_{2}(f(x), f(y))$.

Proposition 14. Let $L$ be an involutive complete residuated lattice, $\left(X, R_{1}\right),\left(Y, R_{2}\right)$ fuzzy approximation spaces, and $f$ a bijective Z-homomorphism, then the following assertions hold:

(1) $R_{1}$ is serial if and only if $R_{2}$ is serial;

(2) $R_{1}$ is reflexive if and only if $R_{2}$ is reflexive;

(3) $R_{1}$ is symmetric if and only if $R_{2}$ is symmetric;

(4) $R_{1}$ is transitive if and only if $R_{2}$ is transitive.

Proof. It is easy to verify from Theorem 10(3).

\section{Approximation Subspaces}

In this section, we introduce the fuzzy approximation subspaces. Let $(X, R)$ be fuzzy approximation space and $R$ a fuzzy equivalence relation, we firstly investigate some properties of $R$-definable fuzzy sets.

Lemma 15. Let $(X, R)$ be fuzzy approximation space and $R a$ fuzzy equivalence relation. Then for every $A \in L^{X}$,

(1) $\bar{R}(A)=A \Leftrightarrow R(x, y) \leq A(y) \rightarrow A(x)$, for every $x, y \in X$

(2) $\underline{R}(A)=A \Leftrightarrow R(x, y) \leq A(x) \rightarrow A(y)$, for every $x, y \in X$

(3) $A$ is a R-definable fuzzy set if and only if $R(x, y) \leq$ $A(x) \leftrightarrow A(y)$, for every $x, y \in X$.

Proof. (1) If $\bar{R}(A)=A$, then $\bar{R}(A)(x)=\bigvee_{y \in X} R(x, y) * A(y)=$ $A(x)$ for every $x \in X$. So for every $y \in X$, we have $R(x, y) *$ $A(y) \leq A(x)$. And this is equivalent to $R(x, y) \leq A(y) \rightarrow$ $A(x)$ for every $y \in X$.

Conversely, for every $x, y \in X, R(x, y) \leq A(y) \rightarrow A(x)$ implies that $\bar{R}(A)(x) \leq A(x)$ for every $x \in X$. Since $R$ is reflexive, we have $A \leq \bar{R}(A)$.

(2) It could be proved similarly as in (1).

(3) It is immediately a deduction of (1) and (2).

Lemma 16. Let $(X, R)$ be fuzzy approximation space and $R a$ fuzzy equivalence relation. If $A, B$ are $R$-definable fuzzy sets, then $A \wedge B, A \vee B, A * B, \neg A$ are $R$-definable fuzzy sets. $R$ definable fuzzy sets of $X$ are closed under arbitrary intersection and union.

Proof. Assume that $A, B$ are $R$-definable fuzzy sets as follows:

$$
\begin{aligned}
&(A \wedge B)(x) \longleftrightarrow(A \wedge B)(y) \\
&=((A \wedge B)(x) \longrightarrow(A \wedge B)(y)) \\
& \wedge((A \wedge B)(y) \longrightarrow(A \wedge B)(x)) \\
& \geq(A(x) \longleftrightarrow A(y)) \wedge(B(x) \longleftrightarrow B(y)) \\
& \geq R(x, y) .
\end{aligned}
$$

According to Lemma 15, we have $A \wedge B$ which is $R$-definable fuzzy set. The claim that $A \vee B, A * B, \neg A$ are $R$-definable fuzzy sets could be proved similarly.

Lemma 17. Let $(X, R)$ be fuzzy approximation space and $R a$ fuzzy equivalence relation. Then every fuzzy equivalence class $R_{z}$ is $R$-definable fuzzy set for $z \in X$. Moreover, $R(x, y)=$ $\bigwedge_{z \in X} R_{z}(x) \leftrightarrow R_{z}(y)=\bigwedge_{A \in \mathscr{C}} A(x) \leftrightarrow A(y)$, where $\mathscr{C}$ is the set of $R$-definable fuzzy sets.

Proof. Since $R$ is transitive, for every $x, y, z \in X$, we have $R(x, y) * R(z, x) \leq R(z, y)$ and $R(y, x) * R(z, y) \leq R(z, x)$; that is, $R(x, y) \leq R(z, x) \rightarrow R(z, y)$ and $R(y, x) \leq R(z, y) \rightarrow$ $R(z, x)$. Because $R$ is symmetric, we have $R(x, y) \leq R(z, x) \leftrightarrow$ $R(z, y)$. Therefore, $R(x, y) \leq \bigwedge_{z \in X} R(z, x) \leftrightarrow R(z, y)$. 
Obviously, $\bigwedge_{z \in X} R(z, x) \leftrightarrow R(z, y) \leq 1 \rightarrow R(x, y)=$ $R(x, y)$. So we have $R(x, y)=\bigwedge_{z \in X} R_{z}(x) \leftrightarrow R_{z}(y) \geq$ $\bigwedge_{A \in \mathscr{C}} A(x) \leftrightarrow A(y)$ since every fuzzy equivalence is a $R$ definable fuzzy set. For every $A \in \mathscr{C}, R(x, y) \leq A(x) \leftrightarrow A(y)$. Hence, $R(x, y)=\bigwedge_{A \in \mathscr{C}} A(x) \leftrightarrow A(y)$.

We propose the fuzzy approximation subspaces induced by fuzzy sets and investigate some of their properties. Let $(X, R)$ be a fuzzy approximation space, $R$ a fuzzy equivalence relation, and $S \in L^{X}$. We define $\left.R\right|_{S} \in L^{X \times X}$, where $\left.R\right|_{S}(x, y)=$ $R(x, y) \wedge(s(x) \leftrightarrow s(y))$ for every $x, y \in X$. Obviously, $\left.R\right|_{S}$ is a fuzzy equivalence relation on $X$.

Definition 18. Let $(X, R)$ be fuzzy approximation space, $R$ an equivalence relation, and $S \in L^{X}$. If $\bar{R}(A)=\overline{\left.R\right|_{S}}(A)$ and $\underline{R}(A)=\left.R\right|_{S}(A)$ for every $A \in L^{X},\left(X,\left.R\right|_{S}\right)$ is called a fuzzy approximation subspace of $(X, R)$.

The next theorem characterizes the correspondence between $R$-definable fuzzy sets and fuzzy approximation subspaces.

Theorem 19. Let $(X, R)$ be fuzzy approximation space, $R$ a fuzzy equivalence relation, and $S \in L^{X}$. Then the following is equivalent:

(1) $S$ is a R-definable fuzzy set;

(2) $\bar{R}(A)=\overline{\left.R\right|_{S}}(A)$ for every $A \in L^{X}$;

(3) $\underline{R}(A)=\left.R\right|_{S}(A)$ for every $A \in L^{X}$;

(4) $\left(X,\left.R\right|_{S}\right)$ is fuzzy approximation subspace of $(X, R)$.

Proof. $(1) \Leftrightarrow(2)$. Since $S$ is a $R$-definable fuzzy set, we have $R(x, y) \leq S(x) \leftrightarrow S(y)$. So $\bar{R}(A)=\overline{\left.R\right|_{S}}(A)$ for every $A \in L^{X}$.

Conversely, $\bar{R}(A)=\overline{\left.R\right|_{S}}(A)$ for every $A \in L^{X}$. We assume that $A=1_{y}$, for all $y \in X$, then $\bar{R}\left(1_{y}\right)(x)=\overline{\left.R\right|_{S}}\left(1_{y}\right)(x)$ for every $x \in X$, that is, $R(x, y)=\left.R\right|_{S}(x, y)$. Therefore, $R(x, y) \leq$ $S(x) \leftrightarrow S(y)$. That is to say, $S$ is a $R$-definable fuzzy set.

$(1) \Leftrightarrow(3)$. We only prove that $\underline{R}(A)=\left.R\right|_{S}(A)$ for every $A \epsilon$ $L^{X}$ which implies that $S$ is a $R$-definable fuzzy set. Assume that $A=R_{y}$, for all $y \in X$, we have $\underline{R}\left(R_{y}\right)(x)=\underline{\left.R\right|_{S}}\left(R_{y}\right)(x)$ for every $x \in X$; that is, $R(x, y)=\left.R\right|_{S}(x, y)$. Hence, $S$ is a $R$-definable fuzzy set.

$(1) \Leftrightarrow(4)$. It is obvious from the verification of $(1) \Leftrightarrow(2)$, $(1) \Leftrightarrow(3)$, and Definition 18 .

We could construct fuzzy approximation subspaces via given ones. Let $(X, R)$ be fuzzy approximation space and $R$ an equivalence relation. Then the union, intersection, and negation of the fuzzy approximation subspaces are defined as follows. Let $A, B \in L^{X}$,

$$
\begin{gathered}
\left(X,\left.R\right|_{A}\right) \cap\left(X,\left.R\right|_{B}\right)=\left(X,\left.\left.R\right|_{A} \cap R\right|_{B}\right), \\
\left(X,\left.R\right|_{A}\right) \cup\left(X,\left.R\right|_{B}\right)=\left(X,\left.\left.R\right|_{A} \cup R\right|_{B}\right), \\
\neg\left(X,\left.R\right|_{A}\right)=\left(X,\left.\neg R\right|_{A}\right) .
\end{gathered}
$$

Proposition 20. Let $(X, R)$ be fuzzy approximation space, $R$ an equivalence relation, and $A, B, A_{i} \in L^{X}, i \in I$ are $R$-definable fuzzy sets. Then the following assertions hold.
(1) $\left.\left.R\right|_{A} \cap R\right|_{B}=\left.R\right|_{A \cap B},\left.\left.R\right|_{A} \cup R\right|_{B}=\left.R\right|_{A \cup B},\left.\neg R\right|_{A}=\left.R\right|_{\neg A}$, $\left.\bigcap_{i \in I} R\right|_{A_{i}}=\left.R\right|_{\bigcap_{i \in I} A_{i}},\left.\bigcup_{i \in I} R\right|_{A_{i}}=\left.R\right|_{\bigcup_{i \in I} A_{i}}$;

(2) $\left(X,\left.R\right|_{A}\right) \cap\left(X,\left.R\right|_{B}\right),\left(X,\left.R\right|_{A}\right) \cup\left(X,\left.R\right|_{B}\right), \neg\left(X,\left.R\right|_{A}\right)$, $\left(X,\left.\bigcup_{i \in I} R\right|_{A_{i}}\right),\left(X,\left.\bigcap_{i \in I} R\right|_{A_{i}}\right)$ are fuzzy approximation subspaces of $(X, R)$. Moreover, the set of all fuzzy approximation subspaces of $(X, R)$ is a complete lattice.

Proof. (1) Since $A, B$ are $R$-definable fuzzy sets, $A \cap B$ is $R$ definable fuzzy set. So we have $R(x, y) \leq A(x) \leftrightarrow A(y)$, $R(x, y) \leq B(x) \leftrightarrow B(y), R(x, y) \leq(A \cap B)(x) \leftrightarrow(A \cap B)(y)$. Hence, $\left.\left.R\right|_{A} \cap R\right|_{B}=\left.R\right|_{A \cap B}$. Similarly, the equations in (1) could be proved.

(2) According to (1) and Theorem 19, it is easy to verify that $\emptyset$ and $X$ are $R$-definable fuzzy set. Therefore, the set of all fuzzy approximation subspaces of $(X, R)$ is a complete lattice.

\section{Homomorphisms between Fuzzy Rough Approximation Spaces}

In this section, we proposed the homomorphisms between two fuzzy approximation spaces based on Frames. Let $\left(X, R_{1}\right)$, $\left(Y, R_{2}\right)$ be fuzzy approximation spaces and $f: X \rightarrow Y$ a map. We define the fuzzy forward operators $\widetilde{f}_{*} \rightarrow \tilde{f}^{*} \rightarrow, \tilde{f}^{\rightarrow}$ : $L^{X} \rightarrow L^{Y}$ as follows. For every $A \in L^{X}, a \in Y$,

$$
\begin{gathered}
\tilde{f}_{*}^{\rightarrow}(A)(a)=\bigvee_{x \in X} A(x) \wedge R_{2}(a, f(x)), \\
\tilde{f}^{* \rightarrow}(A)(a)=\bigvee_{x \in X} A(x) \wedge R_{2}(f(x), a), \\
\tilde{f}^{\rightarrow}(A)(a)=\bigvee_{x \in X} A(x) \wedge R_{2}(a, f(x)) \wedge R_{2}(f(x), a) .
\end{gathered}
$$

And we define the fuzzy backward operators $\widetilde{f}_{*}^{\leftarrow}, \widetilde{f}^{* \leftarrow}, \widetilde{f}^{\leftarrow}$ : $L^{Y} \rightarrow L^{X}$ as follows. For every $B \in L^{Y}, x \in X$,

$$
\begin{gathered}
\tilde{f}_{*}^{\leftarrow}(B)(x)=\bigvee_{y \in X} B(f(y)) \wedge R_{1}(x, y), \\
\tilde{f}^{* \leftarrow}(B)(x)=\bigvee_{y \in X} B(f(y)) \wedge R_{1}(y, x), \\
\tilde{f}^{\leftarrow}(B)(x)=\bigvee_{y \in X} B(f(y)) \wedge R_{1}(x, y) \wedge R_{1}(y, x) .
\end{gathered}
$$

Let $R$ be a crisp binary relation on a set $X$, for any $A, B \subseteq X$; we use the notation $A R=\{b \mid b \in X, \exists a \in A$ such that $(a, b) \in R\}$ and $R B=\{a \mid a \in X, \exists b \in B$ such that $(a, b) \in R\}$.

When $L=\{0,1\}, R_{1}, R_{2}$ are crisp relations on $X, Y$, respectively, and $A \subseteq X, B \subseteq Y$, then

$$
\begin{gathered}
\tilde{f}_{*}^{\rightarrow}\left(\chi_{A}\right)(a)=1 \Longleftrightarrow a \in R_{2}(f(A)), \\
\tilde{f}^{* \rightarrow}\left(\chi_{A}\right)(a)=1 \Longleftrightarrow a \in(f(A)) R_{2}, \\
\tilde{f}^{\rightarrow}\left(\chi_{A}\right)(a)=1 \Longleftrightarrow a \in R_{2}(f(A)) \cap(f(A)) R_{2} .
\end{gathered}
$$

So $\tilde{f}_{*} \rightarrow, \tilde{f}^{*} \rightarrow, \tilde{f}^{\rightarrow}$ are defined as generalizations of $R_{2}(f(A)),(f(A)) R_{2}, R_{2}(f(A)) \cap(f(A)) R_{2} ; \tilde{f}_{*}^{\leftarrow}, \tilde{f}^{* \leftarrow}, \tilde{f}^{\leftarrow}$ 
are generalizations of preimage operators of $R_{1}\left(f^{-1}(B)\right)$, $\left(f^{-1}(B)\right) R_{1}, R_{1}\left(f^{-1}(B)\right) \cap\left(f^{-1}(B)\right) R_{1}$.

Next, we introduce homomorphisms between fuzzy approximation spaces according to the fuzzy forward operators.

Definition 21. Let $\left(X, R_{1}\right),\left(Y, R_{2}\right)$ be fuzzy approximation spaces and $f: X \rightarrow Y$ a map. Then $f$ is called an upper homomorphism if it satisfies $\widetilde{f}_{*} \rightarrow\left(\overline{R_{1}}(A)\right) \subseteq \overline{R_{2}}\left(\widetilde{f}_{*} \rightarrow(A)\right)$, for every $A \in L^{X} ; f$ is called a lower homomorphism if it satisfies $\tilde{f}_{*} \rightarrow\left(R_{1}(A)\right) \subseteq \underline{R_{2}}\left(\tilde{f}_{*} \rightarrow(A)\right)$, for every $A \in L^{X} ; f$ is called a homomorphism between fuzzy approximation space if it is both fuzzy upper and fuzzy lower homomorphism.

Remark 22. We can also define homomorphisms between fuzzy approximation spaces according to $\tilde{f}^{*} \rightarrow, \tilde{f}^{\rightarrow}$. The three kinds of homomorphisms are the same when $R_{2}$ is symmetric. And in the paper, we only discuss the homomorphisms in Definition 21. Most of their properties could be generalized to the other two kinds.

Theorem 23. Let $\left(X, R_{1}\right),\left(Y, R_{2}\right)$ be fuzzy approximation spaces and $f: X \rightarrow Y$ a map. When $R_{2}$ is reflexive and transitive, $f$ is an upper homomorphism if and only if $f$ is relationpreserving.

Proof. For every $x, y \in X$,

$$
\begin{aligned}
\tilde{f}_{*}^{\rightarrow} & \left(\overline{R_{1}}\left(1_{y}\right)\right)(f(x)) \\
& =\bigvee_{z \in X} \overline{R_{1}}\left(1_{y}\right)(z) \wedge R_{2}(f(x), f(z)) \\
& =\bigvee_{z \in X}\left(\bigvee_{u \in X} R_{1}(z, u) \wedge 1_{y}(u)\right) \wedge R_{2}(f(x), f(z)) \\
& =\bigvee_{z \in X} R_{1}(z, y) \wedge R_{2}(f(x), f(z)) ; \\
\overline{R_{2}} & \left(\tilde{f}_{*}^{\rightarrow}\left(1_{y}\right)\right)(f(x)) \\
& =\bigvee_{a \in Y} R_{2}(f(x), a) \wedge\left(\bigvee_{z \in X} 1_{y}(z) \wedge R_{2}(a, f(z))\right) \\
& =\bigvee_{a \in Y} R_{2}(f(x), a) \wedge R_{2}(a, f(y)) .
\end{aligned}
$$

Since $f$ is an upper homomorphism and $R_{2}$ is reflexive, transitive, we have $R_{1}(x, y) \leq R_{2}(f(x), f(y))$; that is, $f$ is relation-preserving.

On the other hand, for every $A \in L^{X}, a \in Y$,

$$
\begin{aligned}
\tilde{f}_{*} & \left(\overline{R_{1}}(A)\right)(a) \\
& =\bigvee_{x \in X}\left(\bigvee_{y \in X} R_{1}(x, y) \wedge A(y)\right) \wedge R_{2}(a, f(x)),
\end{aligned}
$$

$$
\begin{aligned}
\overline{R_{2}}\left(\tilde{f}_{*} \rightarrow(A)\right)(a) \\
\quad=\bigvee_{b \in Y} R_{2}(a, b) \wedge\left(\bigvee_{z \in X} A(z) \wedge R_{2}(b, f(z))\right) .
\end{aligned}
$$

Since $f:\left(X, R_{1}\right) \rightarrow\left(Y, R_{2}\right)$ is a relation-preserving map, we have $R_{1}(x, y) \leq R_{2}(f(x), f(y))$, for every $x, y \in X$. So,

$$
\begin{aligned}
& R_{1}(x, y) \wedge A(y) \wedge R_{2}(a, f(x)) \\
& \quad \leq R_{2}(f(x), f(y)) \wedge A(y) \wedge R_{2}(a, f(x)) \\
& \quad \leq \bigvee_{b \in Y} R_{2}(a, b) \wedge\left(\bigvee_{z \in X} A(z) \wedge R_{2}(b, f(z))\right) .
\end{aligned}
$$

Therefore, $\widetilde{f}_{*} \rightarrow\left(\overline{R_{1}}(A)\right) \subseteq \overline{R_{2}}\left(\widetilde{f}_{*} \rightarrow(A)\right)$.

Corollary 24. Let $\left(X, R_{1}\right),\left(Y, R_{2}\right)$ be fuzzy approximation spaces and $f: X \rightarrow Y$ a map. When $R_{2}$ is reflexive and transitive, $f$ is an upper homomorphism if and only if $\tilde{f}_{*} \rightarrow\left(\overline{R_{1}}\left(1_{x}\right)\right) \subseteq$ $\overline{R_{2}}\left(\widetilde{f}_{*} \rightarrow\left(1_{x}\right)\right)$ for every $x \in X$.

Proof. The claim is easily derived from Theorem 23.

Corollary 25. Let $\left(X, R_{1}\right),\left(Y, R_{2}\right)$ be fuzzy approximation spaces, $R_{2}$ reflexive and transitive, then $f: X \rightarrow Y$ is an upper Z-homomorphism if and only if it is an upper homomorphism.

Proof. The claim is easily derived from Theorems 6 and 23 .

Proposition 26. Let $\left(X, R_{1}\right),\left(Y, R_{2}\right)$ be fuzzy approximation spaces.

(1) If $f: X \rightarrow Y$ is a lower homomorphism, $R_{1}$, $R_{2}$ are reflexive and transitive, then $\tilde{f}_{*} \rightarrow\left(R_{1}\left(R_{1 x}\right)\right)=$ $\underline{R_{2}}\left(\widetilde{f}_{*} \rightarrow\left(R_{1 x}\right)\right)$.

(2) If $f: X \rightarrow Y$ is an upper homomorphism and $R_{1}$, $R_{2}$ are fuzzy equivalence relations on $X, Y$, respectively, then $\widetilde{f}_{*}^{\rightarrow}\left(\overline{R_{1}}\left(R_{1 x}\right)\right)=\overline{R_{2}}\left(\widetilde{f}_{*} \rightarrow\left(R_{1 x}\right)\right)$.

Proof. (1) For every $x \in X, a \in Y, R_{1}, R_{2}$ is reflexive and transitive as follows:

$$
\begin{aligned}
\tilde{f}_{*} & \left(\underline{R_{1}}\left(R_{1 x}\right)\right)(a) \\
& =\bigvee_{z \in X}\left(\bigwedge_{u \in X} R_{1}(z, u) \longrightarrow R_{1 x}(u)\right) \wedge R_{2}(a, f(z)) \\
& =\bigvee_{z \in X} R_{1}(x, z) \wedge R_{2}(a, f(z)), \\
\underline{R_{2}} & \left(\widetilde{f}_{*}^{\rightarrow}\left(R_{1 x}\right)\right)(a) \\
& =\bigwedge_{b \in Y} R_{2}(a, b) \longrightarrow\left(\bigvee_{y \in X} R_{1}(x, y) \wedge R_{2}(b, f(y))\right) .
\end{aligned}
$$

Since $f$ is a lower homomorphism, we have $\bigvee_{z \in X} R_{1}(x, z) \wedge$ $R_{2}(a, f(z)) \leq \bigwedge_{b \in Y} R_{2}(a, b) \rightarrow\left(\bigvee_{y \in X} R_{1}(x, y) \wedge R_{2}(b\right.$, $f(y))) \leq \bigvee_{z \in X} R_{1}(x, z) \wedge R_{2}(a, f(z))$. Therefore, $\widetilde{f}_{*}^{\rightarrow}\left(\underline{R_{1}}\left(R_{1 x}\right)\right)=\underline{R_{2}}\left(\widetilde{f}_{*}^{\rightarrow}\left(R_{1 x}\right)\right)$. 
(2) Since $f$ is an upper homomorphism, we have $\tilde{f}_{*}^{\rightarrow}\left(R_{1 x}\right)=R_{2 f(x)}$ for every $x \in X$. For every $a \in Y$,

$$
\begin{gathered}
\widetilde{f}_{*}^{\rightarrow}\left(\overline{R_{1}}\left(R_{1 x}\right)\right)(a)=\bigvee_{y \in X} R_{1}(x, y) \wedge R_{2}(a, f(y)), \\
\overline{R_{2}}\left(\widetilde{f}_{*}^{\rightarrow}\left(R_{1 x}\right)\right)(a)=\bigvee_{b \in Y} R_{2}(a, b) \wedge R_{2 f(x)}(b)=R_{2}(f(x), a) .
\end{gathered}
$$

Since $f$ is an upper homomorphism, $\tilde{f}_{*} \rightarrow\left(\overline{R_{1}}\left(R_{1 x}\right)\right) \subseteq$ $\overline{R_{2}}\left(\widetilde{f}_{*} \rightarrow\left(R_{1 x}\right)\right)$. The converse is obvious.

Corollary 27. Let $\left(X, R_{1}\right),\left(Y, R_{2}\right)$ be fuzzy approximation spaces, $f: X \rightarrow Y$ a bijective map, and $R_{1}, R_{2}$ fuzzy equivalence relations on $X, Y$, respectively. If $f$ and $f^{-1}$ are both upper homomorphisms, then $R_{1}(x, y)=R_{2}(f(x), f(y))$ for every $x, y \in X$. Moreover, $\widetilde{f}_{*} \rightarrow\left(\overline{R_{1}}(A)\right)=\overline{R_{2}}\left(\widetilde{f}_{*} \rightarrow(A)\right)$ for every $A \in$ $L^{X}$.

Proof. The claim is followed from Proposition 26. Since $f$ is bijective, for every $A \in L^{X}, \widetilde{f}_{*} \rightarrow\left(\overline{R_{1}}(A)\right)=\overline{R_{2}}\left(\widetilde{f}_{*} \rightarrow(A)\right)$ in terms of (20), Theorem 23.

Corollary 28. Let $\left(X, R_{1}\right),\left(Y, R_{2}\right)$ be fuzzy approximation spaces and $f: X \rightarrow Y$ a map, $R_{1}, R_{2}$ are fuzzy equivalence relations on $X, Y$, respectively. If $f$ is a relation isomorphism, then $\tilde{f}_{*} \rightarrow(A)$ is $R_{2}$-definable set when $A$ is $R_{1}$-definable set, for every $A \in L^{X}$.

Proof. Since $f$ is relation isomorphism, $f$ is bijective and $R_{1}(x, y)=R_{2}(f(x), f(y))$ for every $x, y \in X$. From a direct computation, we obtain that $f$ is a homomorphism between $\left(X, R_{1}\right),\left(Y, R_{2}\right)$. So $\tilde{f}_{*} \rightarrow\left(\overline{R_{1}}(A)\right)=\overline{R_{2}}\left(\tilde{f}_{*} \rightarrow(A)\right)$ for every $A \in$ $L^{X}$. Assume that $A$ is a $R_{1}$-definable fuzzy set, then $\overline{R_{1}}(A)=$ $R_{1}(A)$. Hence $\overline{R_{2}}\left(\tilde{f}_{*} \rightarrow(A)\right)=\tilde{f}_{*} \rightarrow\left(\overline{R_{1}}(A)\right)=\tilde{f}_{*} \rightarrow\left(R_{1}(A)\right) \subseteq$ $\overline{R_{2}}\left(\tilde{f}_{*} \rightarrow(A)\right)$, because $f$ is a homomorphism. The converse is obvious, since $R_{2}$ is a fuzzy equivalence relation.

Let $\left(X, R_{1}\right)$ be a fuzzy approximation space and $R$ a fuzzy equivalence relation on $X$. We define a fuzzy relation $\mathscr{R}_{1}$ on the quotient set $X / R$ via

$$
\mathscr{R}_{1}\left(R_{x}, R_{y}\right)=R \circ R_{1} \circ R(x, y)
$$

for every $x, y \in X$.

First, we show that $\mathscr{R}_{1}: X / R \times X / R \rightarrow L$ is a map. For every $x_{i}, y_{i} \in X, i=1,2$ such that $R\left(x_{1}, x_{2}\right)=1, R\left(y_{1}, y_{2}\right)=$ 1 , we have

$$
\begin{aligned}
\mathscr{R}_{1}\left(R_{x_{1}}, R_{y_{1}}\right) & =R \circ R_{1} \circ R\left(x_{1}, y_{1}\right) \\
& =\bigvee_{a \in X} \bigvee_{b \in X} R\left(x_{1}, a\right) \wedge R_{1}(a, b) \wedge R\left(b, y_{1}\right) \\
& =\bigvee_{a \in X} \bigvee_{b \in X} R\left(x_{2}, a\right) \wedge R_{1}(a, b) \wedge R\left(b, y_{2}\right) \\
& =R \circ R_{1} \circ R\left(x_{2}, y_{2}\right) \\
& =\mathscr{R}_{1}\left(R_{x_{2}}, R_{y_{2}}\right) .
\end{aligned}
$$

Proposition 29. Let $\left(X, R_{1}\right)$ be a fuzzy approximation space and $R$ a fuzzy equivalence relation on $X$. $\mathscr{R}_{1}$ is the fuzzy relation defined by (25). Then the following assertions hold:

(1) If $R_{1}$ is reflexive, then $\mathscr{R}_{1}$ is reflexive;

(2) If $R_{1}$ is symmetric, then $\mathscr{R}_{1}$ is symmetric.

Proof. The claim is easy to verify from (25).

If $R_{1}$ is transitive, we could not obtain that $\mathscr{R}_{1}$ is transitive in general. Moreover, even $R_{1}$ is a fuzzy equivalence relation; $\mathscr{R}_{1}$ is not transitive in general. In the following, our discussion is limited within the scope of fuzzy equivalence relations. Let $\left(X, R_{1}\right),\left(Y, R_{2}\right)$ be fuzzy approximation spaces and $f:\left(X, R_{1}\right) \rightarrow\left(Y, R_{2}\right)$ a surjective homomorphism. We define a fuzzy equivalence relation $k_{f}$ on $X$, for every $x, y \in X$, by

$$
k_{f}(x, y)=\bigwedge_{a \in Y} R_{2}(f(x), a) \longleftrightarrow R_{2}(f(y), a) .
$$

Obviously, $k_{f}$ is a fuzzy equivalence relation on $X$ and $k_{f}(x, y)=R_{2}(f(x), f(y))$. For convenience, we let $R=k_{f}$; then $\mathscr{R}_{1}\left(R_{x}, R_{y}\right)=k_{f}(x, y)$ is a fuzzy equivalence relation on $X / R$.

Since the homomorphisms of fuzzy approximation spaces play an important role in data compression and information transmission, so next we introduce the quotient homomorphism.

Proposition 30. Let $\left(X, R_{1}\right),\left(Y, R_{2}\right)$ be fuzzy approximation spaces, where $R_{i}, i=1,2$ are fuzzy equivalence relations. If $f:\left(X, R_{1}\right) \rightarrow\left(Y, R_{2}\right)$ is a surjective homomorphism, then the quotient map $\eta:\left(X, R_{1}\right) \rightarrow\left(X / k_{f}, \mathscr{R}_{1}\right)$ is a homomorphism.

Proof. For every $A \in L^{X}, x_{0} \in X$,

$$
\begin{aligned}
\tilde{\eta}_{*} \rightarrow & \left.\underline{R_{1}}(A)\right)\left(R_{x_{0}}\right) \\
= & \bigvee_{y \in X}\left(\bigwedge_{z \in X} R_{1}(y, z) \longrightarrow A(z)\right) \wedge \mathscr{R}_{1}\left(R_{x_{0}}, R_{y}\right) \\
= & \left.\tilde{f}_{*} \rightarrow \underline{R_{1}}(A)\right)\left(f\left(x_{0}\right)\right) ; \\
\underline{\mathscr{R}_{1}} & \left(\tilde{\eta}_{*}^{\rightarrow}(A)\right)\left(R_{x_{0}}\right) \\
= & \bigwedge_{y \in X} \mathscr{R}_{1}\left(R_{x_{0}}, R_{y}\right) \longrightarrow\left(\bigvee_{x \in X} A(x) \wedge \mathscr{R}_{1}\left(R_{y}, R_{x}\right)\right) \\
& =\underline{R_{2}}\left(\tilde{f}_{*}^{\rightarrow}(A)\right)\left(f\left(x_{0}\right)\right) .
\end{aligned}
$$

Since $f$ is a homomorphism, we have $\tilde{\eta}_{*}^{\rightarrow}\left(R_{1}(A)\right) \subseteq$ $\mathscr{R}_{1}\left(\widetilde{\eta}_{*} \rightarrow(A)\right)$; that is, $\eta$ is a lower homomorphism. Similarly, we could prove that $\eta$ is an upper homomorphism.

Proposition 31. Let $\left(X, R_{1}\right),\left(Y, R_{2}\right)$ be fuzzy approximation spaces, where $R_{i}, i=1,2$ are fuzzy equivalence relations. If $f:\left(X, R_{1}\right) \rightarrow\left(Y, R_{2}\right)$ is a surjective homomorphism and $R_{2}$ is 
a fuzzy equality, then $h:\left(X / k_{f}, \mathscr{R}_{1}\right) \rightarrow\left(Y, R_{2}\right)$ is a bijective homomorphism, where $h\left(\left(k_{f}\right)_{x}\right)=f(x)$ for every $x \in X$.

Corollary 32. Let $\left(X, R_{1}\right)$ be a fuzzy approximation space and $R_{1}, R$ fuzzy equivalence relations on $X$. If $R \leq R_{1}$, then the quotient map $\eta:\left(X, R_{1}\right) \rightarrow\left(X / R, \mathscr{R}_{1}\right)$ is a homomorphism.

\section{Conclusions}

We have discussed two kinds of homomorphisms between fuzzy approximation spaces based on complete residuated lattices in this paper. The notion of homomorphism established the communication of two universes, so we can deal with one fuzzy approximation space in terms of information of another one. We also have investigated fuzzy approximation subspaces and their correspondence with definable fuzzy sets. Under some particular conditions, the factor sets of fuzzy approximations spaces are also discussed. In the future, we will intend to study the application of homomorphisms of fuzzy approximation spaces. Moreover, we will investigate the communication between the fuzzy covering rough sets.

\section{Conflict of Interests}

The author declares that there is no conflict of interests regarding the publication of this paper.

\section{Acknowledgment}

The author would like to thank editor Kazutake Komori and the anonymous referees for their valuable suggestions in improving this paper.

\section{References}

[1] Z. Pawlak, "Rough sets," International Journal of Computer and Information Sciences, vol. 11, no. 5, pp. 341-356, 1982.

[2] Z. Pawlak, Rough Sets: Theoretical Aspects of Reasoning About Data, Kluwer Academic Publishers, Boston, Mass, USA, 1991.

[3] D. Boixader, J. Jacas, and J. Recasens, "Upper and lower approximations of fuzzy sets," International Journal of General Systems, vol. 29, no. 4, pp. 555-568, 2000.

[4] M. De Cock, C. Cornelis, and E. Kerre, "Fuzzy rough sets: beyond the obvious," in Proceedings of the IEEE International Conference on Fuzzy Systems, vol. 1, pp. 103-108, July 2004.

[5] D. Dubois and H. Prade, "Rough fuzzy sets and fuzzy rough sets," International Journal of General Systems, vol. 17, pp. 191208, 1990.

[6] N. N. Morsi and M. M. Yakout, "Axiomatics for fuzzy rough sets," Fuzzy Sets and Systems, vol. 100, no. 1-3, pp. 327-342, 1998.

[7] A. M. Radzikowska and E. E. Kerre, "A comparative study of fuzzy rough sets," Fuzzy Sets and Systems, vol. 126, no. 2, pp. 137$155,2002$.

[8] H. Thiele, "On axiomatic characterization of fuzzy approximation operators III. The fuzzy diamond and fuzzy box based cases," in Proceedings of the 10th IEEE International Conference on Fuzzy Systems, vol. 2, pp. 1148-1151, December 2001.

[9] W.-Z. Wu, J.-S. Mi, and W.-X. Zhang, "Generalized fuzzy rough sets," Information Sciences, vol. 151, pp. 263-282, 2003.
[10] W.-Z. Wu, Y. Leung, and J.-S. Mi, "On characterizations of $(I, T)$-fuzzy rough approximation operators," Fuzzy Sets and Systems, vol. 154, no. 1, pp. 76-102, 2005.

[11] Y. Y. Yao, "Constructive and algebraic methods of the theory of rough sets," Information Sciences, vol. 109, no. 1-4, pp. 21-47, 1998.

[12] A. M. Radzikowska and E. E. Kerre, "Fuzzy rough sets based on residuated lattices," in Transactions on Rough Sets II, vol. 3135 of Lecture Notes in Computer Science, pp. 278-296, 2004.

[13] Y.-H. She and G.-J. Wang, "An axiomatic approach of fuzzy rough sets based on residuated lattices," Computers \& Mathematics with Applications, vol. 58, no. 1, pp. 189-201, 2009.

[14] J. W. Graymala-Busse, "Algebraic properties of knowledge representation systems," in Proceedings of the ACM SIGART International Symposium on Methodologies for Intelligent Systems, pp. 432-440, Knoxville, Tennessee, 1986.

[15] C. Wang, C. Wu, D. Chen, Q. Hu, and C. Wu, "Communicating between information systems," Information Sciences, vol. 178, no. 16, pp. 3228-3239, 2008.

[16] C. Wang, D. Chen, and L. Zhu, "Homomorphisms between fuzzy information systems," Applied Mathematics Letters, vol. 22, no. 7, pp. 1045-1050, 2009.

[17] P. Zhu and Q. Wen, "Some improved results on communication between information systems," Information Sciences, vol. 180, no. 18, pp. 3521-3531, 2010.

[18] P. Zhu and Q. Wen, "Homomorphisms between fuzzy information systems revisited," Applied Mathematics Letters, vol. 24, no. 9, pp. 1548-1553, 2011.

[19] K. Blount and C. Tsinakis, "The structure of residuated lattices," International Journal of Algebra and Computation, vol. 13, no. 4, pp. 437-461, 2003.

[20] M. Ward and R. P. Dilworth, "Residuated lattices," Transactions of the American Mathematical Society, vol. 45, no. 3, pp. 335-354, 1939.

[21] J. A. Goguen, “L-fuzzy sets," Journal of Mathematical Analysis and Applications, vol. 18, pp. 145-174, 1967.

[22] W. Bandler and L. J. Kohout, "On the general theory of relational morphisms," International Journal of General Systems, vol. 13, no. 1, pp. 47-66, 1987.

[23] M. K. Chakraborty and M. Das, "Studies in fuzzy relations over fuzzy subsets," Fuzzy Sets and Systems, vol. 9, no. 1, pp. 79-89, 1983.

[24] M. K. Chakraborty and M. Das, “On fuzzy equivalence I," Fuzzy Sets and Systems, vol. 11, no. 1-3, pp. 163-183, 1983.

[25] M. K. Chakraborty and M. Das, "On fuzzy equivalence II," Fuzzy Sets and Systems, vol. 11, no. 1-3, pp. 287-297, 1983.

[26] M. K. Chakraborty and S. Sarkar, "Fuzzy antisymmetry and order," Fuzzy Sets and Systems, vol. 21, no. 2, pp. 169-182, 1987.

[27] L. A. Zadeh, "Similarity relations and fuzzy orderings," Information Sciences, vol. 3, pp. 177-200, 1971.

[28] M. Ćirić, J. Ignjatović, and S. Bogdanović, "Fuzzy equivalence relations and their equivalence classes," Fuzzy Sets and Systems, vol. 158, no. 12, pp. 1295-1313, 2007. 


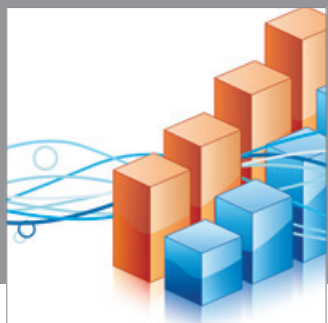

Advances in

Operations Research

mansans

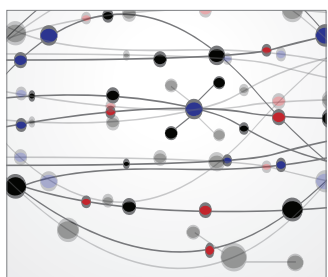

The Scientific World Journal
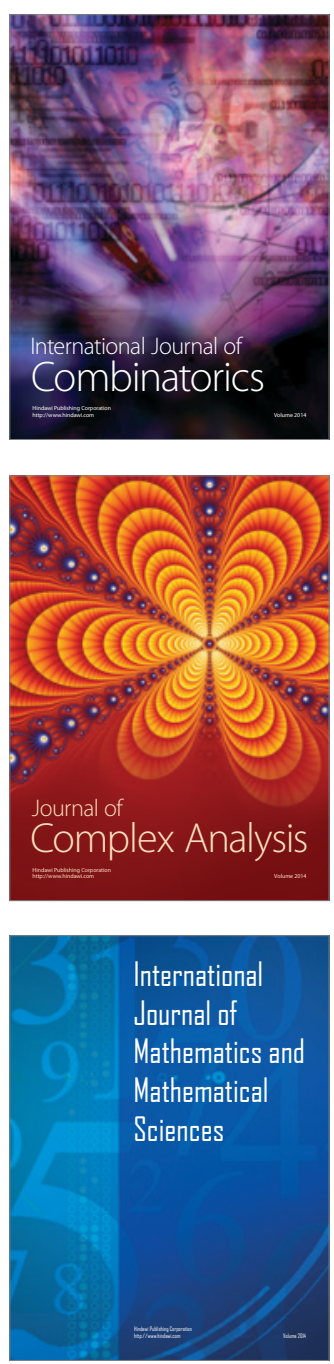
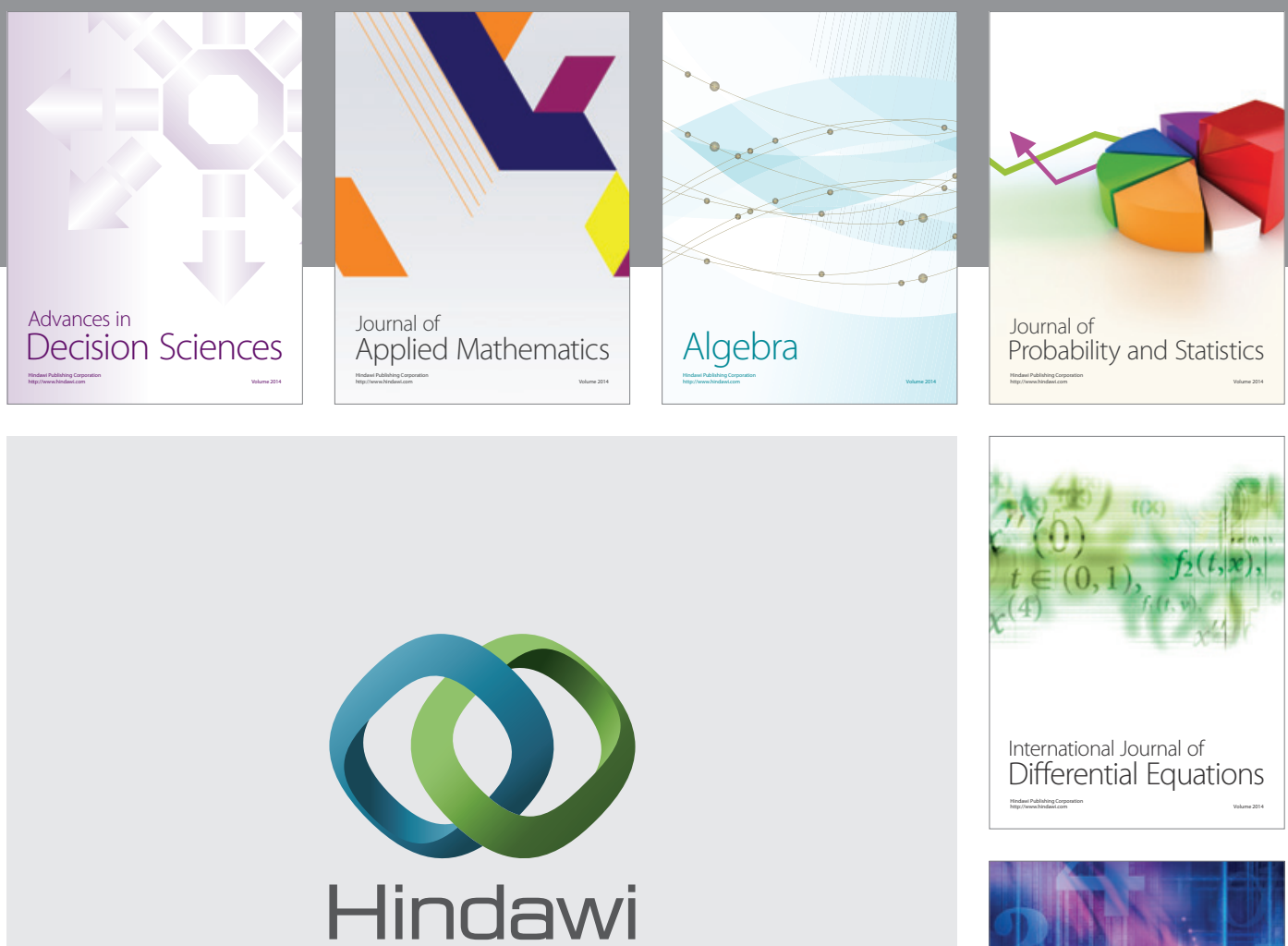

Submit your manuscripts at http://www.hindawi.com
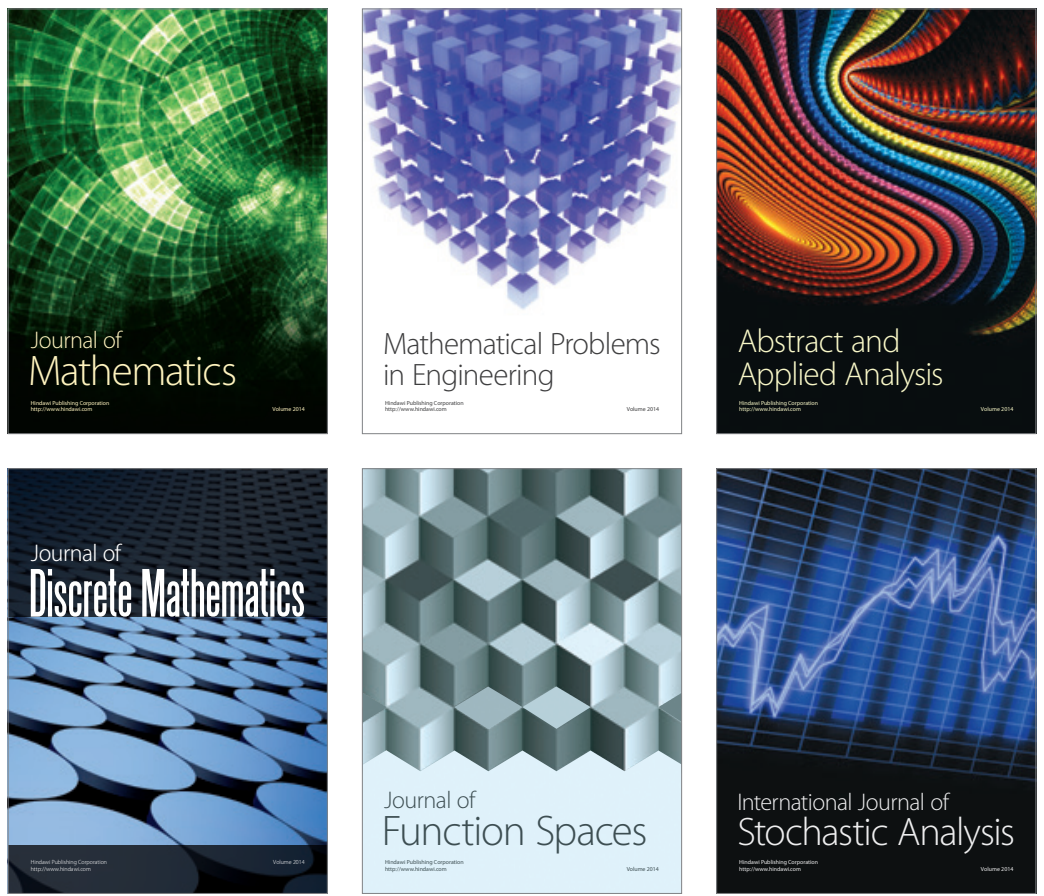

Journal of

Function Spaces

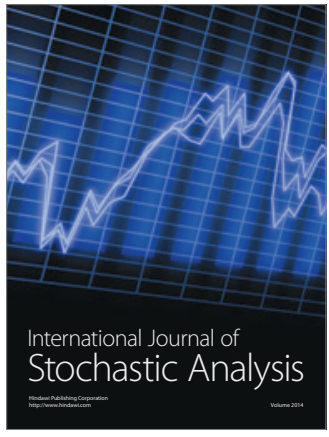

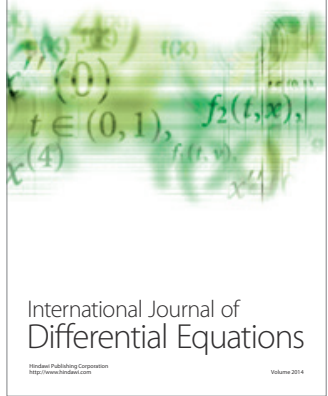
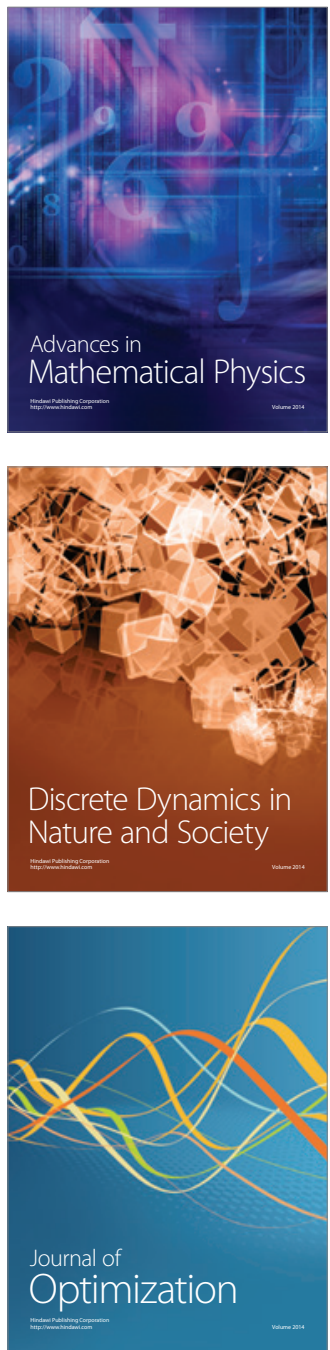